\title{
Adaptação cardiovascular no Teste de Caminhada dos Seis Minutos em pacientes com DPOC: estudo transversal
}

\section{Cardiovascular adaptation in the six-minute walk test in COPD patients: transversal study}

\section{José Reinaldo Oliveira Silva', Julia Ribeiro Santana ${ }^{2}$, Carolina Correia da Silva ${ }^{3}$, Daniel França Seixas Simões ${ }^{4}$, Aquiles Assunção Camelier ${ }^{5}$, Fernanda Warken Rosa Camelier ${ }^{6}$}

'Autor para correspondência. Universidade do Estado da Bahia. Salvador, Bahia, Brasil. ORCID: 0000-0003-4617-9328. reinaldooliveira17@hotmail.com 2Universidade do Estado da Bahia. Salvador, Bahia, Brasil. ORCID: 0000-0002-7571-2083. julia_ribeiro.s@hotmail.com ${ }^{3}$ Universidade do Estado da Bahia. Salvador, Bahia, Brasil. ORCID: 0000-0002-4944-7538. carolcorreia0504@gmail.com ${ }_{4}^{4}$ Pulsar Salvador. Salvador, Bahia, Brasil. ORCID: 0000-0002-4002-3704. francasdaniel@gmail.com ${ }^{5}$ Universidade do Estado da Bahia. Escola Bahiana de Medicina e Saúde Pública. Salvador, Bahia, Brasil. ORCID: 0000-0001-5410-5180. aquilescamelier@yahoo.com.br ¿Universidade do Estado da Bahia. Salvador, Bahia, Brasil. ORCID: 0000-0003-2540-0142. fcamelier@uneb.br

RESUMO | OBJETIVO: Avaliar o comportamento da adaptação cardiovascular e da saturação periférica de oxigênio em indivíduos com DPOC submetidos no teste de caminhada dos seis minutos (TC6). MATERIAL E MÉTODOS: Trata-se de um estudo de corte transversal, que foram incluídas pessoas com diagnóstico de DPOC, confirmado pela espirometria e de ambos os sexos. A magnitude de sintomas foi avaliada pela escala de dispneia Medical Research Council (MRC) e questionário COPD Assessment Test (CAT). Aplicou-se o TC6 para avaliar a tolerância ao esforço. Para mensurar a frequência cardíaca máxima (FC máx) prevista para a idade foram utilizadas equações específicas para população brasileira. RESULTADOS: Avaliou-se 34 indivíduos com DPOC, 20 (58,8\%) homens; relação VEF1/CVF foi $56,7 \%$ $\pm 10,2 \%$ pós broncodilatador (BD). Quatorze $14(41,2 \%)$ indivíduos apresentaram impacto clínico moderado; $16(47,2 \%)$ dos avaliados apresentou grau 2 na escala do MRC. As médias das distâncias percorridas no primeiro e segundo TC6 foram 383,5 $\pm 13,6 ; 408,6 \pm 85,7$ metros, correspondendo a 70, 75\%; $75,10 \%$ em relação ao valor previsto $(p=0,001)$. As médias da FC máx ao final do primeiro e segundo TC6, foram 94,1 $\pm 21,9$; $92,3 \pm 17,9 \mathrm{bpm}$ e a $\mathrm{FC}$ pós percentual da FC máx prevista pré e pós TC6 foram $61,1 \% \pm 17,7 \% ; 59,7 \% \pm 21,5 \%$ e 14 $(41,2 \%)$ pacientes apresentaram dessaturação de $\mathrm{O}_{2}$ no primeiro TC6; $9(26,5 \%)$ no segundo teste. CONCLUSÕES: Pacientes com DPOC, apresentam aumento da FC identificado pelo esforço submáximo, por meio do percentual da FC max. Indivíduos com maior comprometimento da função pulmonar apresentaram dessaturação de $\mathrm{O}_{2}$.

PALAVRAS-CHAVE: Doença Pulmonar Obstrutiva Crônica. Frequência cardíaca. Dessaturação de oxigênio. Teste de Caminhada dos Seis Minutos.
ABSTRACT I OBJECTIVE: To evaluate the behavior of cardiovascular adaptation and peripheral oxygen saturation in individuals with COPD submitted to the six-minute walk test (6MWT). MATERIAL AND METHODS: It was performed a descriptive study with person with a diagnosis of COPD confirmed by spirometry of both sexes. The Medical Research Council (MRC) dyspnea scale and the COPD Assessment Test (CAT) questionnaire were used to assess the magnitude of symptoms. The 6MWT was used to assess effort tolerance. To measure the maximum heart rate (HRmax) predicted for age, specific equations were used for the Brazilian population. RESULTS: 34 individuals with COPD were evaluated, 20 (58.8\%) men; FEVI / FVC ratio was $56.7 \%$ $\pm 10.2 \%$ after BD. Fourteen $(41.2 \%)$ were classified as grade 2 and were classified as grade 2 (MRC scale). The means of the distances covered in the first and second TC6 were $383.5 \pm$ $13.6 ; 408.6 \pm 85.7$ meters, corresponding to $70.7 \% ; 75.1 \%$ in relation to the predicted value $(p=0.001)$. The mean maximum heart rate at the end of the first and second 6MWT were 94.1 $\pm 21.9 ; 92.3 \pm 17.9 \mathrm{bpm}$ and the heart rate post-percentage of the predicted maximum heart rate before and after the 6MWT were $61.1 \% \pm 17.7 \% ; 59.7 \% \pm 21.5 \%$ and $14(41.2 \%)$ patients presented $\mathrm{O}_{2}$ desaturation on the $6 \mathrm{MWT} ; 9(26.5 \%)$ in the second test. CONCLUSIONS: Patients with COPD throughout the 6MWT show increased heart rate and $\mathrm{O}_{2}$ desaturation in exercise activity.

KEYWORDS: Chronic Obstructive Pulmonary Disease. Heart rate. Oxygen desaturation. Six-minute Walk Test. 


\section{Introduçâo}

A Doença Pulmonar Obstrutiva Crônica (DPOC) é uma afecção frequente, prevenível e tratável. É caracterizada por obstrução crônica e persistente das vias aéreas de pequeno e médio calibre, geralmente progressiva. Está associada a uma reação inflamatória pulmonar causada principalmente pela exposição à fumaça de cigarro, exposição ocupacional e combustão de biomassa. O quadro clínico da doença é caracterizado por dispneia, tosse e expectoração, exacerbações e redução da capacidade de exercício $^{1,2}$. Além das alterações no sistema respiratório, há repercussões sistêmicas como a fraqueza muscular, disfunção dos membros inferiores (MMII) e fadiga, fazendo com que o indivíduo apresente diminuição da tolerância ao esforço físico que impactam diretamente na capacidade funcional ${ }^{3}$.

A avaliação funcional torna-se indispensável, tanto para determinar o estado funcional quanto para direcionamento das ações terapêuticas, incluindo a reabilitação pulmonar ${ }^{4}$. Dessa forma, o Teste de Caminhada dos Seis Minutos (TC6), visa avaliar a capacidade funcional do indivíduo sendo muito utilizado por ser de baixo custo, rápido, seguro e não necessita de equipamento de alta tecnologia, além de correlacionar bem com as atividades diárias e é considerado um preditor de mortalidade em pacientes com DPOC. À distância percorrida durante os seis minutos é o parâmetro mais estudado do teste, pois permite avaliar e classificar a gravidade e prognóstico da doença que está relacionado com desempenho no mesmo ${ }^{5}$.

Ademais, o TC6 reflete mais significativamente 0 comportamento da variação da FC e a dessaturação de $\mathrm{O}_{2}$ que é induzida pelo esforço submáximo em indivíduos com DPOC do que, os demais testes funcionais existente na literatura. Inúmeros fatores contribuem para que pessoas com DPOC tenham o comportamento alterado da FC, dentre estes a hipoxemia, hiperinsuflação dinâmica e inflamação sistêmica que poderiam afetar a resposta submáxima da FC ao exercício com consequências adversas no prognóstico das doenças ${ }^{6}$.

A dessaturação periférica de oxigênio que é induzida pela caminhada dos seis minutos, fornece informações clínicas importantes e que está associada com a redução da atividade de vida diária (AVDs) do indivíduo, demonstrando um declínio mais acentuado do volume expiratório forçado no primeiro segundo (VEF1) e a piora do prognóstico da doença ${ }^{7}$.

É sabido que devido às manifestações da doença pulmonar, as pessoas com DPOC geralmente relatam dispnéia e fadiga em MMII com frequência, quando estão realizando suas tarefas diárias 1. Uma vez que esses indivíduos encontram-se limitados para execução das atividades físicas de vida diária, torna-se essencial conhecer o nível do comprometimento funcional. Sendo assim, o TC6 é de fundamental importância na avaliação das pessoas com DPOC, pois aponta os fatores limitantes do exercício. Portanto, o objetivo principal do presente estudo é avaliar o comportamento da adaptação cardiovascular e da $\mathrm{SpO}_{2}$ em indivíduos com DPOC submetidos à caminhada dos seis minutos.

\section{Material e metodos}

Foi conduzido um estudo descritivo, de corte transversal, no qual participaram pessoas portadoras de DPOC em acompanhamento pelo Programa Assistencial a Pessoa com Doenças Respiratórias Crônicas, um programa de extensão universitária, vinculado do Curso de Fisioterapia do Departamento de Ciências da Vida (DCV) da Universidade do Estado da Bahia - UNEB, Salvador - Bahia, que atende pacientes cadastrados pelo Sistema Único de Saúde (SUS), no período de fevereiro a junho de 2018. Foram incluídos no estudo os pacientes com diagnóstico de DPOC, estáveis de acordo com as diretrizes da Global Iniciative for Chronic Obstructive Lung Disease' (GOLD, 2017), de ambos os sexos e que tenham realizado espirometria revelando uma relação volume expiratório forçado no primeiro segundo e capacidade vital forçada $\left(\mathrm{VEF}_{1} / \mathrm{CVF}\right)<$ 0,70 após broncodilatador (BD).

Os critérios de exclusão foram: 1 - qualquer condição clínica, detectada por meio de avaliação clínica por um médico, que impedisse a realização do TC6 (incluindo hipertensão arterial não controlada, angina instável, infarto agudo do miocárdio nos últimos três meses, insuficiência cardíaca descompensada ou problemas ortopédicos que impedissem 0 
desempenho no teste; 2- Incapacidade técnica na captação dos sinais de monitorização da $\mathrm{SpO}_{2}$ e da FC no oxímetro de pulso durante a realização do TC6; 3-Retirada do Termo de consentimento livre e esclarecido.

As fontes de dados foram primária e secundária e a coleta de dados foi realizada no Laboratório de Fisiologia do Exercício, DCV/UNEB, Salvador-Bahia. Os indivíduos foram submetidos a uma avaliação por profissionais treinados, capacitados e com ampla experiência em pesquisa que ocorreu em um único dia, no qual foram verificadas as características sociodemográficas: idade em anos; sexo (masculino e feminino); cor (branco, mulato, negro, amarelo); peso $(\mathrm{Kg})$; altura (metro); índice de massa corporal (IMC) $\left(\mathrm{kg} / \mathrm{m}^{2}\right)$; estado civil (casado/união estável, solteiro, viúvo) e as clínicas como: classificação da doença pela espirometria, o prognóstico, a magnitude de sintomas respiratórias, além da realização do TC6, por duas vezes, com intervalo de até 30 minutos entre eles. Os dados de função pulmonar recente foram obtidos no prontuário do paciente do ambulatório de referência.

Os pacientes foram separados em grupos conforme a classificação de gravidade da limitação ao fluxo de ar, determinados pela GOLD 2017', avaliando parâmetros como a capacidade vital forçada (CVF), o volume expiratório forçado no primeiro segundo (VEF1) e a relação VEF1/CVF, identificando a limitação do fluxo aéreo, definida principalmente pela da relação VEF1/CVF abaixo de $70 \%$ pós-broncodilatador (BD)'.

Para avaliação dos sintomas de magnitude respiratória foi utilizado o questionário COPD Assessment Test (CAT), validado para a língua portuguesa/ Brasil, que é composto de oito itens, denominados tosse, catarro, aperto no peito, falta de ar, limitações nas atividades domiciliares, confiança em sair de casa, sono e energia. Os resultados variam de acordo com a faixa dos escores obtidos, classificados da seguinte forma em relação ao impacto clínico: 6- 10 pontos, leve; 11-20, moderado; $21-30$, grave; e 31-40; muito grave e variando o escore entre 0 (melhor) e 40 (pior) ${ }^{8}$.

Na avaliação da dispneia foi utilizada escala de dispneia Medical Research Council (MRC) que é um instrumento que avalia $\circ$ grau de dispnéia desde uma atividade física extenuante até uma simples realização das atividades da vida diária. Esta escala é dividida em cinco graus: o grau 0 indica que o paciente refere falta de ar quando realiza atividade física intensa como correr, nadar, praticar esporte. O grau 1 indica que o paciente refere falta de ar quando caminha de maneira apressada no plano ou quando sobe uma superfície elevada. $O$ grau 2 refere que o paciente anda mais devagar do que pessoas da mesma idade pela falta de ar; ou quando o paciente ao caminhar no plano, no próprio passo, tem que parar para respirar. $\bigcirc$ grau 3 indica que 0 paciente, após andar alguns metros ou alguns minutos no plano, tem que parar para respirar. $O$ grau 4 refere que o paciente sente falta de ar quando este realiza uma simples atividade de vida diária (AVD) ou quando esta falta de ar já chegou a um momento de impedi-lo de sair de casa?.

Para avaliar os comprometimentos sistêmicos da DPOC que refletem nas limitações para executar as atividades de vida diária (AVD), foi utilizado o índice BODE. Esse instrumento é considerado um sistema de graduação multidimensional composto por quatro domínios, são eles: o grau de comprometimento da função pulmonar (VEF1) pós-uso do broncodilatador; a percepção de dispneia pela escala $M R C_{\text {; }}$ índice de massa corporal (IMC) e capacidade de exercício (TC6) pela distância percorrida em metros. $O$ índice BODE compreende uma pontuação que varia de zero a dez, dividida em quatro quartis. Sendo que o quartil 1 a pontuação varia de 0 a 2; quartil 2, de 3 a 4; quartil 3, de 5 a 6 e quartil 4, de 7 a 10. A partir dessa divisão é possível classificar funcionalmente o indivíduo portador da doença, desta forma, quanto maior a pontuação, maior a limitação nas $A V D$ s e também o risco de mortalidade dessa população ${ }^{10}$.

Para aplicação do TC6 foram seguidas as orientações da ATS ${ }^{11}$ que recomenda realizado o teste por duas vezes, em uma superfície plana, com piso antiderrapante sob supervisão de uma equipe treinada e capacitada. É um teste simples, bem tolerado e reprodutível, requerendo equipamentos de baixo custo $^{5}$. $O$ voluntário foi avaliado pelo pesquisador antes e após o teste, através da frequência cardía$\mathrm{ca}(\mathrm{FC})$, saturação periférica de oxigênio $\left(\mathrm{SpO}_{2}\right)$ por meio de um oxímetro de pulso portátil (Rossmax ${ }^{\circledR}$, 
modelo Palpus1 SA210), pressão arterial sistólica e diastólica mensuradas com o indivíduo na posição sentada e com um esfigmomanômetro aneróide (Premiun®,G-TECH), frequência respiratória (FR), dispnéia e fadiga de membros inferiores por meio da escala de Borg Modificada. Ao término do teste, os parâmetros foram aferidos imediatamente. Antes do teste considerado para a pesquisa, foi orientado a realizar o pré- teste que auxiliou na compreensão. Houve um intervalo de 10 minutos para repouso, e em seguida o pesquisador cronometrou - tempo para realização do teste. Para predizer a distância percorrida durante o tempo estabelecido foi utilizado valor de referência para adulto saudáveis, ambos os sexos, baseado em um estudo descrito por Britto et al. ${ }^{12}$. Para calcular a frequência cardíaca máxima prevista para a idade foram utilizadas equações para a população brasileira, onde FC $\max =211-(0,87 *$ idade $)$ para homens e FC $\max =212-(0,89 *$ idade $)$ para mulheres 13. Para avaliar a comportamento da adaptação cardiovascular, foram estudados $\triangle \mathrm{FC}=(\mathrm{FC}$ pico $-\mathrm{FC}$ repouso $) 14$ e $F C$ reserva $=(F C$ pico $-F C$ repouso $) /$ (220 - idade - FC repouso) x 10015. Com relação à queda percentual de $\mathrm{O}_{2^{\prime}}$ na prática clínica é considerada a dessaturação periférica de $\mathrm{O}_{2}$ no esforço quando a $\mathrm{SpO}_{2}$ ocorre uma variação de $4 \%{ }^{16}$.

O estudo foi realizado conforme amostra de conveniência, sendo que a amostra de 34 indivíduos foi capaz de proporcionar, em termos de precisão, uma capacidade de deteç̧ão de uma variação de 8 mmHg na pressão arterial sistólica ou diastólica, ou 5 bpm na frequência cardíaca, baseados em dados da literatura4 e de tabela de cálculo amostral descritas em Hulley SB et al ${ }^{17}$.
O plano de análise do estudo foi realizado por meio de planilha de dados estruturada no programa Excel e a análise no SPSS (v.22.0). Os dados foram expressos em medida de tendência central, dispersão e proporções, com relação avaliação de normalidade dos dados foi utilizado o teste Shapiro-Wilk e a comparação do desempenho pré e pós TC6 foi feita através do teste $-T$ para amostras em pares. A significância estatística foi investigada com a análise de variância para grupos normalmente distribuídos e $p<0,05$ foi considerado estatisticamente significativo.

O estudo seguiu a Resolução $N^{\circ} 466 / 2012$ do Conselho Nacional de Saúde do Ministério da Saúde e foi aprovado pelo Comitê de Ética e Pesquisa da Universidade do Estado da Bahia, CAAE 81687517.6.0000.0057. Todos os participantes foram voluntários e concordaram em ler e assinar o Termo de Consentimento Livre e Esclarecido.

\section{Resultados}

Trinta e quatro indivíduos com diagnóstico de DPOC foram examinados de acordo com os critérios de elegibilidade e incluídos na análise final do estudo (Figura 1), sendo $20(58,8 \%)$ eram do sexo masculino e $19(55,9 \%)$ tinham menos ou igual 60 anos. Observou-se que $31(92,7 \%)$ indivíduos foram classificados como tendo cor da pele preta e 21 (61,8\%) eram casados ou viviam em união estável e com relação ao nível de escolaridade $17(50,0 \%)$ dos avaliados possuem ensino fundamental completo. Em relação ao Índice de Massa Corpórea (IMC), destacou-se que $17(50,0 \%)$ são eutróficos (Tabela 1$)$. 


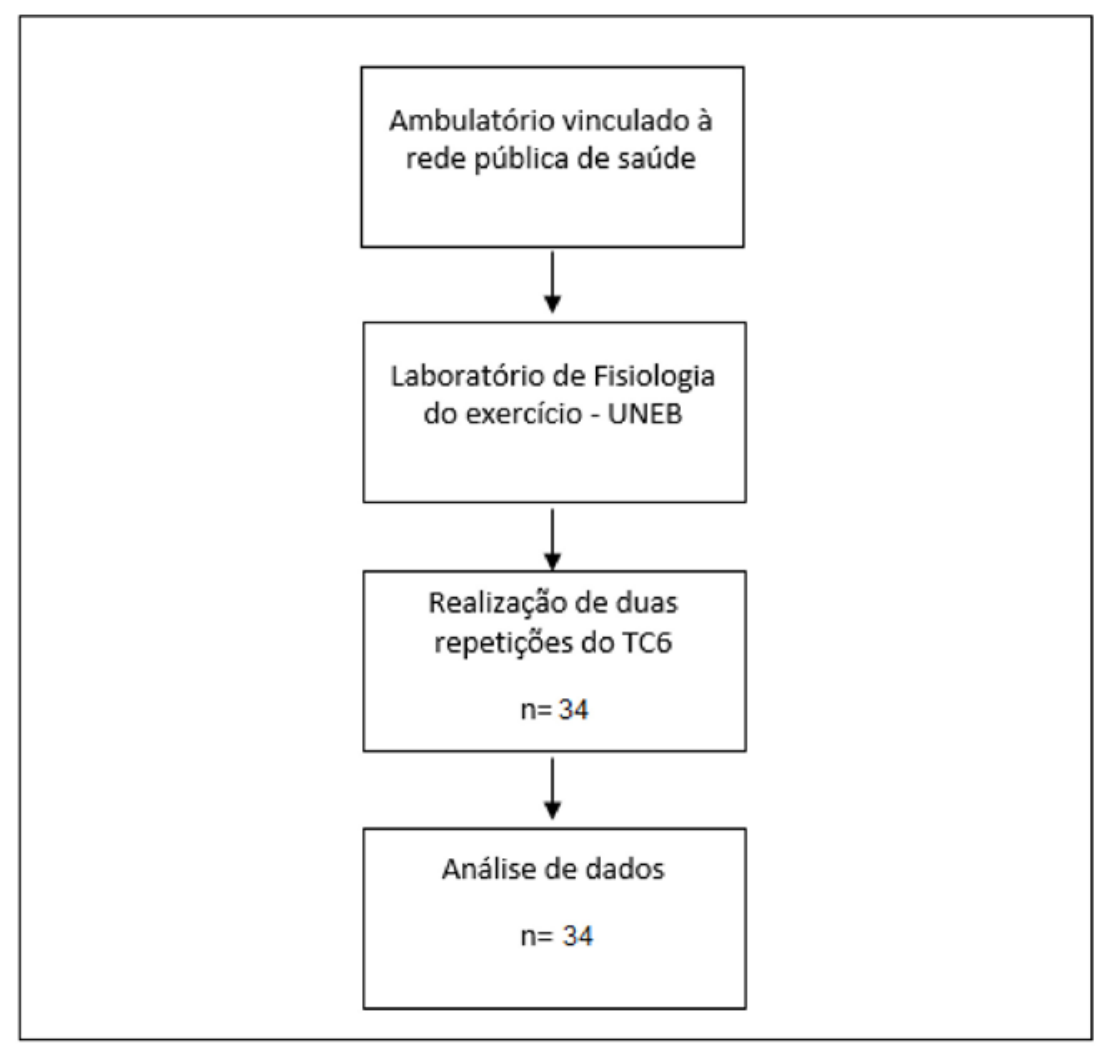

Tabela 1. Características sociodemográficas dos pacientes com DPOC,

atendidos no Laboratório de Fisiologia do Exercício de uma universidade pública, Salvador, BA, Brasil, 2018 ( $n=34)$

\begin{tabular}{clcc}
\hline \multicolumn{1}{c}{ Característica } & $\mathbf{n}$ & $\%$ \\
\hline Sexo & Masculino & 20 & 58,8 \\
& Feminino & 14 & 41,2 \\
Idade & & & \\
$\quad \leq 60$ anos & 19 & 55,9 \\
$\quad>60$ anos & 15 & 44,1 \\
Estado Civil & & \\
$\quad$ Casado/ União Estável & 21 & 61,8 \\
$\quad$ Solteiro & 13 & 38,2 \\
Nível de Escolaridade & & 11,8 \\
$\quad$ Sem instrução & 4 & 50,0 \\
$\quad$ Ensino Fundamental & 17 & 35,3 \\
$\quad$ Ensino Médio & 12 & 2,9 \\
$\quad$ Ensino Superior & 1 & 92,7 \\
Cor da Pele & & 7,1 \\
$\quad$ Preto & 31 & 11,8 \\
$\quad$ Branco & 3 & 50,0 \\
Estado Nutricional & & 38,2 \\
\hline Baixo peso & 4 & \\
$\quad$ Eutrófico & 17 & \\
Sobrepeso & 13 &
\end{tabular}


Na Tabela 2 encontram-se descritas as características clínicas dos pacientes com DPOC. Notou-se que a função pulmonar apresentou valores médios pós broncodilatador (BD) de, CVF de 66,6\% $\pm 14,6$ do previsto, VEF 1 de $46,5 \% \pm 13,1 \%$ do previsto e a relação VEF1 /CVF foi de $56,7 \% \pm 10,2 \%$. Mais de $70,0 \%$ dos indivíduos foram classificados com grau moderado da doença de acordo com o VEF1 e no índice BODE, $13(38,2 \%)$ pacientes foram classificados no quartil um e dois. Em relação aos sintomas tosse, catarro, aperto no peito, falta de ar, limitações nas atividades domiciliares, confiança em sair de casa, sono e energia avaliados pelo questionário COPD Assessment Test (CAT) $14(41,2 \%)$ apresentaram impacto clínico moderado e grave. Observouse que $16(47,2 \%)$ pacientes possuem limitação na locomoção devido à falta de ar e precisam parar para respirar em algum momento do descolamento (escala do MRC).

Tabela 2. Características clínicas dos pacientes com DPOC,

atendidos no Laboratório de Fisiologia do Exercício de uma universidade pública, Salvador, BA, Brasil, 2018 ( $n=34$ ).

\begin{tabular}{|c|c|c|}
\hline Variável & Média & DP \\
\hline \multicolumn{3}{|l|}{ Função pulmonar } \\
\hline CVFL pósBD & 2,3 & 0,6 \\
\hline VEF,LpósBD & 1,2 & 0,4 \\
\hline CVF\%pósBD & 66,6 & 14,6 \\
\hline $\mathrm{VEF}_{1} \%$ pós BD & 46,5 & 13,1 \\
\hline \multirow[t]{2}{*}{$\mathrm{VEF}_{1} / \mathrm{CVF}$} & 56,7 & 10,2 \\
\hline & $\mathbf{n}$ & $\%$ \\
\hline \multicolumn{3}{|c|}{ Classificação da gravidade da doença (Critérios GOLD) } \\
\hline A & 6 & 17,6 \\
\hline B & 9 & 26,5 \\
\hline $\mathrm{C}$ & 2 & 5,9 \\
\hline $\mathrm{D}$ & 17 & 50,0 \\
\hline \multicolumn{3}{|c|}{ Classificação da gravidade da doença $\left(\mathrm{VEF}_{1}\right)$} \\
\hline Moderado & 24 & 70,6 \\
\hline Grave & 10 & 29,4 \\
\hline \multicolumn{3}{|l|}{ Impacto Clínico } \\
\hline Leve & 6 & 17,6 \\
\hline Moderado & 14 & 41,2 \\
\hline Grave & 14 & 41,2 \\
\hline \multicolumn{3}{|l|}{ Grau de dispneia } \\
\hline Grau 0 & 1 & 2,9 \\
\hline Grau 1 & 6 & 17,6 \\
\hline Grau 2 & 16 & 47,2 \\
\hline Grau 3 & 5 & 14,7 \\
\hline Grau 4 & 6 & 17,6 \\
\hline \multicolumn{3}{|l|}{ Índice de prognóstico BODE } \\
\hline Quartil 1 & 13 & 38,2 \\
\hline Quartil 2 & 13 & 38,2 \\
\hline Quartil 3 & 7 & 20,6 \\
\hline Quartil 4 & 1 & 2,9 \\
\hline
\end{tabular}

CVF L: Capacidade Vital Forçada em Litros; CVF \%: Capacidade Vital Forçada em Porcentagem; VEF 1 \%: Volume Expiratório Forçado no Primeiro Segundo em Porcentagem; VEF, L: Volume Expiratório Forçado no Primeiro Segundo em Litros; BD: Broncodilatador; DP: Desvio Padrão e GOLD: Global Iniciative for Chronic Obstructive Lung Disease. 
As médias das distâncias percorridas no primeiro e segundo TC6 foram, respectivamente, $387,5 \pm 85,6$ e $411,3 \pm 87,3$ metros e os testes não foram interrompidos. Quando comparado o desempenho no TC6 com aquele proposto por Britto et al., ${ }^{12}$ os participantes caminharam $70,75 \%$ metros no primeiro TC6 e 75,10\% metros no segundo teste, quanto ao previsto pela equação de referência da distância percorrida para adultos saudáveis e ambos sexos $(p=<0,001)$. As variáveis analisadas não tiveram diferenças estatisticamente significantes quando comparados os valores pós testes com os basais. Quando comparando o pré e pós-teste, houve aumento estatisticamente significante da frequência cardíaca $(F C)$ frequência respiratória (FR), pressão arterial (PA), percepção da dispneia e do cansaço de membros inferiores, como também diminuição da saturação periférica de oxigênio, sem repercussão clínica deste parâmetro. As médias da variação da FC e a de reserva pré e pós teste foram respectivamente $20,2 \pm 15,8 \mathrm{bpm} ; 18,8 \pm 10,7 \mathrm{bpm} ; 26,2$ $\pm 20,4$ bpm; $24,6 \pm 15,3$ bpm não sendo estatisticamente significantes. Com relação à FC máxima prevista para idade a média foi de 153,7 $\pm 8,8$. Ademais notou-se que as médias da FC máxima ao final do primeiro e segundo TC6, foram 94,1 $\pm 21,9$ e $92,3 \pm 17,9 \mathrm{bpm}(p=0,875)$ e a FC pós percentual da FC máxima prevista pré e pós TC6 foram $61,1 \% \pm 17,7 \%$ e $59,7 \% \pm 21,5 \%$, foi estatisticamente significante $(p<0,05)$. Além disso, a dessaturação periférica de $\mathrm{O}_{2}$ observada no presente estudo foi evidenciada em indivíduos com maior comprometimento da função pulmonar.

Tabela 3. Distância prevista e percorrida com as descrições das variáveis obtidas pré e pós TC6 dos indivíduos com DPOC, atendidos no Laboratório de Fisiologia do Exercício de uma universidade pública, Salvador, BA, Brasil, 2018 ( $n=34)$.

\begin{tabular}{|c|c|c|c|c|c|}
\hline Variáveis & Média/DP & & & & \\
\hline \multirow[t]{2}{*}{$\begin{array}{l}\text { FC máx prevista idade (bpm) } \\
\text { DTC6 prevista }(\mathrm{m})\end{array}$} & $\begin{array}{l}153,7 \pm 8,8 \\
547,7 \pm 45,7\end{array}$ & & & & \\
\hline & TC6 1 & $\mathbf{P}$ & TC6 2 & $\mathbf{p}$ & $\mathbf{p}^{*}$ \\
\hline DTC6 (m) & $387,5 \pm 85,6$ & - & $411,3 \pm 87,3$ & - & 0,001 \\
\hline FC pré (bpm) & $73,7 \pm 11,2$ & & $73,8 \pm 12,6$ & & 0,405 \\
\hline FC pós (bpm) & $94,1 \pm 21,9$ & $<0,001$ & $92,3 \pm 17,9$ & $<0,001$ & 0,875 \\
\hline FC pós $\%$ FC max prevista (bpm) & $61,1 \pm 17,7$ & & $59,7 \pm 21,5$ & & $<0,001$ \\
\hline$\Delta \mathrm{FC}$ pós x pré (bpm) & $20,2 \pm 15,8$ & & $18,8 \pm 10,7$ & & 0,620 \\
\hline $\mathrm{FC}$ reserva (bpm) & $26,2 \pm 20,4$ & & $24,6 \pm 15,3$ & & 0,627 \\
\hline FR pré (ipm) & $19,2 \pm 4,4$ & & $21,8 \pm 10,9$ & & 0,144 \\
\hline FR pós (ipm) & $23,4 \pm 5,4$ & $<0,001$ & $25,8 \pm 18,9$ & 0,023 & 0,472 \\
\hline PAS pré (mmHg) & $127,1 \pm 14,3$ & & $128,1 \pm 16,4$ & & 0,913 \\
\hline PAS pós (mmHg) & $138,2 \pm 22,8$ & 0,003 & $137,3 \pm 26,3$ & 0,026 & 0,833 \\
\hline PAD pré (mmHg) & $83,1 \pm 20,3$ & & $86,1 \pm 14,7$ & & 0,498 \\
\hline PAD pós $(\mathrm{mmHg})$ & $81,5 \pm 20,9$ & 0,697 & $88,1 \pm 12,4$ & 0,489 & 0,069 \\
\hline $\mathrm{SpO}_{2}$ pré (\%) & $95,9 \pm 2,1$ & & $95,3 \pm 2,1$ & & 0,195 \\
\hline $\mathrm{SpO}_{2}$ pós (\%) & $92,4 \pm 4,9$ & $<0,001$ & $91,5 \pm 6,2$ & 0,023 & 0,192 \\
\hline 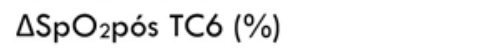 & $-3,4 \pm 4,1$ & & $-3,8 \pm 5,3$ & & $<0,001$ \\
\hline Borg MMll pré & $0,6 \pm 1,1$ & & $0,7 \pm 1,5$ & & 0,705 \\
\hline Borg MMll pós & $2,6 \pm 2,9$ & $<0,001$ & $2,3 \pm 2,7$ & 0,001 & 0,231 \\
\hline Borg dispneia pré & $0,6 \pm 1,1$ & & $0,6 \pm 1,1$ & & 0,951 \\
\hline Borg dispneia pós & $2,5 \pm 2,9$ & $<0,001$ & $2,8 \pm 2,5$ & 0,001 & 0,657 \\
\hline & $\mathbf{n}$ & $\%$ & $\mathbf{n}$ & $\%$ & \\
\hline $\mathrm{DSpO}_{2}$ & 14 & 41,2 & 9 & 26,5 & \\
\hline $\begin{array}{l}\text { * A correlação foi realizada através } \\
\text { o segundo teste; Frequência cardíac } \\
\text { da freqüência cardíaca (variação } \\
\text { (DTC6) no primeiro e segundo TC } \\
\text { frequência respiratória (FR) pré e pó } \\
\text { dos testes, saturação de pulso de o }\end{array}$ & $\begin{array}{l}\text { - T de amostr } \\
\text { a prevista parc } \\
\text { quência cardía } \\
\text { jência cardíac } \\
\text { pressão arteric } \\
\left.\mathrm{SpO}_{2}\right) \text { pré tes }\end{array}$ & $\begin{array}{l}\text { em pares } \\
\text { dade (FC } \\
\text { reserva } \\
\text { (FC) pré } \\
\text { sistólica ( } P \text {, } \\
\text { e ao final }\end{array}$ & $\begin{array}{l}\text { *:Associação do } \\
\text { ax prevista parc } \\
\text { C reserva), des } \\
\text { pós-teste no } \\
\text { ) e diastólica (P } \\
\text { Variação da sa }\end{array}$ & $\begin{array}{l}\text { primeiro } 1 \\
\text { idade), v } \\
\text { mpenho } \\
\text { xercício } \\
\text { AD) pré a } \\
\text { uração pe }\end{array}$ & $\begin{array}{l}\text { C6 com } \\
\text { ariação } \\
\text { o teste } \\
\text { áximo, } \\
\text { ao final } \\
\text { riférica }\end{array}$ \\
\hline $\begin{array}{l}\text { de oxigênio }\left(\triangle \mathrm{SpO}_{2} \text { pós } \mathrm{TC} 6\right) \text {, valor } \\
\text { percepção de dispneia (Borg } \mathrm{D}) \text { pré } \\
\text { pós TC6 }\left(\mathrm{DSpO}_{2}\right) \text {. }\end{array}$ & $\begin{array}{l}\text { a percepção de } \\
\text { teste, Dessaturc }\end{array}$ & $\begin{array}{l}\text { cansaço en } \\
\text { ão saturac }\end{array}$ & membros inferi & res (Borg & WMII) e \\
\hline
\end{tabular}




\section{Discussão}

O presente estudo descreve a adaptação cardiovascular e da troca gasosa durante a realização do TC6 em portadores de DPOC acompanhados ambulatorialmente. $O$ esforço padronizado, de característica submáxima, induzido pelo TC6 promoveu o aumento da $\mathrm{FC}$ e induziu a dessaturação de $\mathrm{O}_{2} \mathrm{em}$ portadores da DPOC. Os participantes assumiram, ao final do TC6, em torno de $60 \%$ da máxima FC prevista, ratificando este comportamento submáximo do exercício assumido. Houve também a queda da $\mathrm{SpO}_{2}$ em média no primeiro e segundo teste respectivamente, $-3,4 \pm 4,1$ e $3,8 \pm 5,3$, 。 que confirma a função da caminhada dos seis minutos em identificar dessaturação da oxihemoglobina durante este teste e corroborando com achados na literatura $^{18-22}$.

Estes achados fisiológicos indicam um determinado nível de exercício assumido que justifica a utilização do TC6 na avaliação da capacidade funcional em indivíduos com enfermidade obstrutiva crônica. Por ser um teste simples, de baixo custo e de fácil manuseio, torna-se viável sua aplicação na prática clínica e principalmente em locais de pouco espaço ${ }^{5}$.

Quando se compara a FC inicial e final no pré e pós TC6, é possível observar aumento significativo na FC ao final do teste. $O$ aumento da FC em indivíduos com DPOC em uma atividade de esforço submáximo pode ser atribuído por diversos aspectos fisiopatológicos: pelo aumento da demanda metabólica; pelo aprisionamento de ar (também denominado de hiperinsuflação dinâmica), que leva ao aumento da pressão intratorácica que, consequentemente, produz redução do volume de ejeção do ventrículo esquerdo e, por sua vez, reflete no aumento da FC (na intenção de manter a elevação do débito cardíaco imposta pelo aumento da demanda metabólica) e que, por sua vez, é mediado pela ativação dos barorreceptores 19. Outros aspectos relacionados à dinâmica cárdiopulmonar na DPOC também justificam a elevação da FC no TC6. Um deles é a estimulação dos receptores de estiramento pulmonar, induzida pelo aumento do volume corrente durante a inspiração, os quais, quando estimulados, produzem cardioaceleração e consequente taquicardia ${ }^{18-20}$.
Ademais, pessoas com DPOC apresentam aumento limitado do volume sistólico que pode ser explicado por vários fatores, dentre eles a diminuição da reserva de pré-carga do ventrículo direito, redução da reserva inotrópica do ventrículo direito, isquemia miocárdica, insuficiência da tricúspide, comprometimento da interação ventricular direita-esquerda e alteração da função diastólica do ventrículo direito ${ }^{20}$. Além disso, o aumento da FC durante a realização do TC6 pode estar relacionado com a incapacidade da elevação do débito cárdico que acaba refletindo a piora do prognóstico da doença ${ }^{18}$. Já foi também demonstrado na literatura que, durante a realização do TC6, pacientes com DPOC terminam adotando um nível de velocidade de caminhada auto determinado, que, de acordo com as alterações fisiopatológicas impostas pela doença, é ajustado de maneira inconsciente para atingir um nível de exercício suportável, submáximo, que, por definição, corresponde à " potência crítica" ou "velocidade crítica de caminhada" descrita por Casas e colaboradores, reproduzindo, desta maneira, uma atividade espontânea de vida diária, e justificando a utilização deste teste como uma maneira de se avaliar a capacidade funcional utilizando uma atividade bastante similar à uma atividade cotidiana ${ }^{21}$.

A dessaturação de $\mathrm{O}_{2}$ em pessoas com DPOC, induzida pelo TC6 neste estudo está justificada pela presença da alteração da relação ventilação - perfusão presente nestes pacientes, incrementada pelo esforço, sendo aspecto fisiopatológico conhecido na DPOC'. Estes aspectos são justificados pela limitação ventilatória, pela desproporção entre a oferta e o consumo de $\mathrm{O}_{2}$, além da inflamação sistêmica e do estresse oxidativo, que acabam afetando a oxigenação muscular periférica ${ }^{22,23}$. Dessa forma, existe a queda significativa nos níveis de oxigênio circulantes a nível tanto sanguíneo quanto muscular, fator que se torna responsável pela estimulação simpáti$\mathrm{ca}$, ocasionando aumento da pressão arterial, da resistência vascular periférica, e, consequentemente, aumentando a percepção da dispneia e a fadiga muscular, reduzindo assim a tolerância na execução de exercícios submáximos por estes pacientes, podendo atuar estes fatores fisiopatológicos presentes tanto isoladamente quanto em conjunto ${ }^{7}$.Estudos prévios mostraram que 0 tempo de dessaturação durante o TC6 é um indicativo da possibilidade de dessaturar durante as atividades cotidianas, culmi- 
nando em hipoxemia severa e necessidade de oxigenoterapia ${ }^{7,12,14}$.

No presente estudo também foi identificada a redução significativa da distância percorrida no TC6 comparada ao valor previsto pela equação para pessoas saudáveis e de ambos os sexos, baseado no estudo de Britto et $a l^{12}$. Os indivíduos com DPOC no estudo caminharam de 70 a $75 \%$ da distância máxima prevista pela idade, fator que poderia ser justificado, além dos aspectos fisiopatológicos descritos, também pela inatividade/sedentarismo, comum nestes pacientes, bem como no estilo de vida adotado, pela baixa frequência e intensidade de esforço durante a realização das atividades de vida diária, fatores sociais, culturais e, principalmente, a desmotivação dessas pessoas no momento da realização das tarefas diárias que está relacionado com a gravidade dos sintomas'.

A percepção da dispnéia e o cansaço em MMIl é uma das principais queixas referidas pelas pessoas com DPOC, que acaba resultando na diminuição da funcionalidade e declínio da independência funcional. Essas informações foram observadas nesses resultados, em que esses sintomas foram capazes de comprometer o desempenho dos voluntários avaliados. A principal explicação para esse resultado seria por causa da condição física, grau de instrução sobre a doença e fatores sociais. Entretanto, alguns trabalhos científicos, demonstram que a sensação de dispneia e cansaço em MMII, são os principais sintomas da intolerância ao esforço ${ }^{14,21,22}$. Esses resultados são importantes para o entendimento de que não só a mecânica pulmonar, mais outros fatores estão envolvido na tolerância ao exercício, bem como, troca gasosa anormal, disfunção cardíaca, disfunção da musculatura esquelética global ${ }^{22-24}$.

Acrescenta-se ainda que a alteração do estado nutricional em pessoas com DPOC é uma complicação frequente nesse público, pois estão relacionados com as repercussões sistêmicas e estresse oxidativo provenientes da doença e além de apresentar perda de massa muscular. No presente estudo ficou evidente que o IMC da amostra estudada se encontra entre os valores normais de referência ${ }^{25,26}$.

Dentre as vantagens do TC6 (apresentado nesse estudo) está a fácil realização do teste na avaliação da capacidade funcional, bem como, demonstrando o comportamento da $\mathrm{FC}$ e da $\mathrm{SpO}_{2}$ em pessoas com DPOC e o baixo custo (pois não necessita de equipamentos de alta tecnologia), além de ser um teste seguro e validado na literatura ${ }^{1,11}$. Outro ponto importante do presente estudo é a capacidade que o TC6 possui para correlacionar-se com as atividades de vida diária, determinando o perfil funcional dos indivíduos com DPOC, conferindo uma aplicação prática e útil.

As limitações da pesquisa referem-se, em primeiro lugar, tanto ao tamanho da amostra que foi por conveniência; quanto à característica dos pacientes, portadores de DPOC já acompanhados ambulatorialmente em serviço universitário público e com sintomas mais intensos e gravidade mais marcada, o que pode conferir limitação, por um lado, na generalização dos resultados principalmente para ambiente de atenção primária onde pacientes com nível mais leve de acometimento possa talvez ser mais comum.

\section{Conclusão}

Pode-se concluir que o TC6 induz um aumento da FC em nível de esforço submáximo, de acordo com - valor previsto para a idade. Pessoas com DPOC também apresentam dessaturação de $O_{2}$ durante a realização do TC6, o que confere na prática a este simples e de baixo custo teste, a capacidade de realizar a avaliação funcional das pessoas com esta doença.

\section{Contribuição dos autores}

Os autores Silva JRO, Camelier AA e Camelier FWR elaboraram a ideia inicial e planejaram o trabalho. Silva JRO, Santana JR, Silva CC e Camelier FWR interpretaram os resultados finais. Silva JRO, Camelier AA e Camelier FWR redigiram o artigo. Silva JRO, Santana JR, Silva CC, França D, Camelier AA e Camelier FWR revisaram sucessivas versões e aprovaram a versão final do artigo.

\section{Conflitos de interesses}

Nenhum conflito financeiro, legal ou político envolvendo terceiros (governo, empresas e fundações privadas, etc.) foi declarado para nenhum aspecto do trabalho submetido (incluindo mas não limitandose a subvenções e financiamentos, conselho consultivo, desenho de estudo, preparação de manuscrito, análise estatística, etc.). 


\section{Referências}

1. Global Initiative for Chronic Obstructive Lung Disease (GOLD). Global Strategy for the Diagnosis, Management, and Prevention of Chronic Obstructive Lung Disease, 2017.

2. Calik-Kutukcu E, Savci S, Saglam M, Varda-Yagli N, Arikan $\mathrm{H}$, Aribas $Z$ et al. A comparison of muscle strength and endurance, exercise capacity, fatigue perception and quality of life in patients with Chronic Obstructive Pulmonary Disease and healthy subjects: a cross-sectional study. BMC Pulm Med. 2014;14:6. doi: 10.1186/1471-2466-14-6

3. Langer D, Hendriks E, Burtin C, Probst V, van der Schans $C$, Paterson $W$ et al. A clinical practice guideline for physiotherapists treating patients with chronic obstructive pulmonary disease based on a systematic review of available evidence. Clin Rehabil. 2009;23(5):445-62. doi: $10.1177 / 0269215509103507$

4. Ozalevli S, Ozden A, Itil O, Akkoclu A. Comparison of the Sit-to-Stand Test with 6 min walk test in patients with Chronic Obstructive Pulmonary Disease. Respir Med. 2007;101(2):286-93. doi: 10.1016/i.rmed.2006.05.007

5. Meriem $M$, Cherif J, Toujani $S$, Ouahchi $Y$, Hmida $A B$, Beji $M$. Sit to stand test and 6 min walking test correlation in patients with chronic obstructive pulmonary disease. 2015;10(4):269-273. doi: 10.4103/1817-1737.165289

6. Richter MJ, Milger K, Tello K, Stille P, Seeger W, Mayer E. Heart rate response during 6-minute walking testing predicts outcome in operable chronic thromboembolic pulmonary hypertension. BMC Pulm Med. 2016;16(1)96. doi: 10.1186/ s1 2890-016-0260-y

7. Moreira MAF, Medeiros GA, Boeno FP, Sanches PRS, Silva Júnior DP, Müller AF. Análise da dessaturação de oxigênio durante o teste de caminhada de seis minutos em pacientes com DPOC. J Bras Pneumol. 2014;40(3):222-228. doi: $10.1590 / \mathrm{S} 1806-37132014000300004$

8. Silva GPF, Morano MTAP, Viana CMS, Magalhaes CBA, Pereira EDB. Portuguese-language version of the COPD Assessment Test: validation for use in Brazil. J Bras Pneumol. 2013;39(4):402-408. doi: 10.1590/S180637132013000400002

9. Bestall JC, Paul EA, Garrod R, Garnham R, Jones PW, Wedzicha JA. Usefulness of the Medical Research Council (MRC) dyspnoea scale as a measure of disability in patients with chronic obstructive pulmonary disease. Thorax. 1999;54(7):581-6.

10. Celli BR, Cote CG, Marin JM, Casanova C, Oca MM, Mendez RA et al. The body-mass index, airflow obstruction, dyspnea, and exercise capacity index in chronic obstructive pulmonary disease. N Engl J Med. 2004;350(10):1005-12. doi: $10.1056 /$ NEJMoa021322
11. Holland $A E$, Spruit MA, Troosters T, Puhan $M A$, Pepin V, Saey D et al. An official European Respiratory Society/American Thoracic Socity technical standard: field walking tests in chronic respiratory disease. Eur Respir J. 2014;44(6):1 428-1446. doi: $10.1183 / 09031936.00150314$

12. Britto RR, Probst VS, Andrade AFD, Samora GAR, Hemandes NA, Marinho PEM et al. Reference equations for the six-minute walk distance based on a Brazilian multicenter study. Braz J Phys Ther. 2013;17(6):556-563. doi: 10.1590/ S1413-35552012005000122

13. Neder JA, Nery LE. Fisiologia Clinica do Exercício Teoria e Pratica. São Paulo: Artes Medicas; 2003.

14. Provencher $S$, Herve $P$, Sitbon $O$, Humbert $M$, Simonneau $G$, Chemla D. Changes in exercise haemodynamics during treatment in pulmonary arterial hypertension. Eur Respir J. 2008;32(2):393-398. doi: 10.1183/09031936.00009008

15. Azarbal B, Hayes SW, Lewin HC, Hachamovitch R, Cohen I, Berman DS. The incremental prognostic value of percentage of heart rate reserve achieved over myocardial perfusion single-photon emission computed tomography in the prediction of cardiac death and all-cause mortality: superiority over 85 $\%$ of maximal age-predicted heart rate. J Am Coll Cardiol. 2004;44(2):423-430. doi: 10.1016/i.jacc.2004.02.060

16. Lama VN, Flaherty KR, Toews GB, Colby TV, Travis WD, Long $Q$ et al. Prognostic value of desaturation during a 6-minute walk test in idiopathic interstitial pneumonia. Am J Respir Crit Care Med. 2003;1 68(9):1084-1090. doi: 10.1164/rccm.200302-2190C

17. Browner WS, Newman TB, Cummings SR, Hulley SB. Estimating sample size and power: the nity-gritty. In: Hulley SB, Cummings SR, Browner WS, Grady D, Hearst N, Newman $T B$, editors. Designing clinical research: an epidemiologic approach. 2nd ed. Baltimore: Ed. Williams \& Wilkins; 2001. P. 65-91.

18.Larsen PD, Tzeng YC, Sin PY, Galletly DC. Respiratory sinus arrhythmia in conscious humans during spontaneous respiration. Respir Physiol Neurobiol. 2010;174(1-2):1 11 -8. doi: 10.1016/i.resp.2010.04.021

19. Marshall JM. Peripheral chemoreceptors and cardiovascular regulation. Physiol Rev. 1994;74(3):543-94. doi: $10.1152 /$ physrev.1994.74.3.543

20. Laskey WK, Ferrari VA, Palevsky HI, Kussmaul WG. Pulmonary artery hemodynamics in primary pulmonary hypertension. J Am Coll Cardiol. 1993;21 (2):406-412.

21. Russo R, lamonti VC, Jardim JR. Intolerância ao exercício no paciente com DPOC. Pneumol Paulista. $2012 ; 26(1)$ :38-41. 
22. Holland AE, Spruit MA, Singh SJ. How to carry out a field walking test in chronic respiratory disease. Breathe. 2015; 11 (2):1 28-139. doi: 10.1183/20734735.021314

23. Poulain $M$, Durand F, Palomba B, Ceugniet $F$, Desplan $J$, Varray $A$ et al. Six minute walk testing is more sensitive than maximal incremental cycle testing for detecting oxygen desaturation in patients with COPD. Chest. 2003; 1 23(5): $1401-7$.

24. Marino DM, Marrara KT, Lorenzo VAP, Jamami M. Teste de caminhada de seis minutos na doença pulmonar obstrutiva crônica com diferentes graus de obstrução. Rev Bras Med. 2007;13(2):103-106. doi: 10.1590/S1517$\underline{86922007000200007}$

25. Hamilton Al, Killian KJ, Summers E, Jones NL. Muscle strength symptom intensity and exercise capacity in patients with cardiorespiratory disorders. Am J Respir Crit Care Med. 1995;1 52(6):2021-2031. doi: 10.1164/ ajrccm.152.6.8520771

26. Schettino CDS, Deus FCC, Gonçalves AAV, Wallace E. Relação entre DPOC e Doença Cardiovascular. Pulmão RJ. 2013;22(2):19-23. 